Christopher Sinke*, Janina Neufeld ${ }^{*}$, Markus Zedler, and Hinderk M. Emrich

Hannover Medical School; Clinic for Psychiatry, Social Psychiatry and Psychotherapy, Germany

${ }^{*}$ C. Sinke and J. Neufeld contributed equally to this paper

\author{
Correspondence to: \\ Janina Neufeld \\ Hannover Medical School; \\ Clinic for Psychiatry, Social Psychiatry and Psychotherapy \\ Carl-Neuberg-Straße 1 \\ 30625 Hannover \\ Germany \\ Email: neufeld.janina@mh-hannover.de \\ Phone: 00495115326658
}

\title{
Synaesthesia: a Conceptualization ('Synthesis'-) Phenomenon. Philosophical and Neurobiological Aspects
}

\begin{abstract}
Neurobiological aspects of synaesthesia are discussed from the perspective of the broader philosophical topic of "syn-aisthesis" and the basic fundamentals of a neuropsychological understanding of perceptual inter-modal integration. Herein, the predominance of conceptualization processes in regard to top-down functions of the brain appears as a prerequisite for perception. Functional Magnet Resonance Imaging (fMRI) data of synaesthetes compared to controls are discussed, providing evidence for the theory that prefrontal and parietal conceptualization processes by themselves exert transmodal functions and thus contain properties of "binding". A partial hyperactivity of such processes in synaesthesia may thus be a causal factor of this condition.
\end{abstract}

Keywords: synaesthesia; syn-aisthesis; unitarity of consciousness; binding; Immanuel Kant; bottom-up and top-down functions; prefrontal and parietal conceptualization. 


\section{Aisthesis and synthesis}

Neurobiology of consciousness represents one of the most challenging research topics in natural science - as regards the relationship between neuronal realities and mental life which takes place within our minds. Herein, one central question is how the different modalities are integrated during the process of "aisthesis" (Greek = perception) in order to give rise to the unified percepts we experience in our mind. This unification process is also termed "synthesis" or "binding". For example, we do not see a car and hear the sound of its engine separately but we perceive a car as an integral object ("Gestalt") (Wertheimer 1938) with visual and acoustical qualities.

At the neuronal level, we know since the elegant pioneering work of Hubel and Wiesel (Hubel and Wiesel 1979) that the primary visual data are analysed step by step by highly specialized neuronal assemblies which detect, e.g., the direction of a movement, a contour, the colour or another quality of retinal excitation/inhibition patterns. This parallel processing of different aspects of sensory data also takes place in other modalities, e.g., in audition (Merzenich and Brugge 1973). While it enables us to analyse parts of the signal, this process results in fragmentation of the sensory data, leading to the famous computer-brain analogy which fits in with our everyday understanding of perception: From a naive point of view, one assumes that outer reality is strictly constituted in precisely that fashion in which it appears to the subject. This is as if it is sufficient to photograph or film outer objects; and subjective experience represents nothing else than the function of a computer which calculates from sensory data outer reality (Fig. 1a). The brain would - in this metaphor - be a pure 'sink', no 'source' of information (and meaning).

Here, one main question arises which has to do with the problem of 'unitarity of consciousness', namely the problem of the so called 'intermodal integration', the 'binding' problem: How does it come that we observe in perception not an addition of elements of percepts but a type of holisms, i.e., 'perceptual gestalts'? The mechanism by which our consciousness integrates different aspects of a perceived object is, as a matter of fact, not yet elucidated.

However, as Immanuel Kant already pointed out, an interpretation of reality is possible only if a leading conceptualization (a 'world model') is applied to the raw material of sensory inputs (Fig. 1b). Before sensory data can be calculated and interpreted, a set of working hypotheses about possible outer realities is required. This is presented philosophically - 
in Kant's 'Critique of Pure Reason' (Kant 1974) - as conceptualization and in psychological terms of the 'accompanying world model' by Prinz (Prinz 2006). Wolfgang Welsch (Welsch 1992) has formulated this referring to Immanuel Kant enunciating "we can cognize of things a priori only what we ourselves have put into them" (Kant, 'Kritik der reinen Vernunft'; quote translation as per Allen W. Wood (1998)) and these are primarily aesthetic preconditions of, namely, the recognition-categories of space and time.'
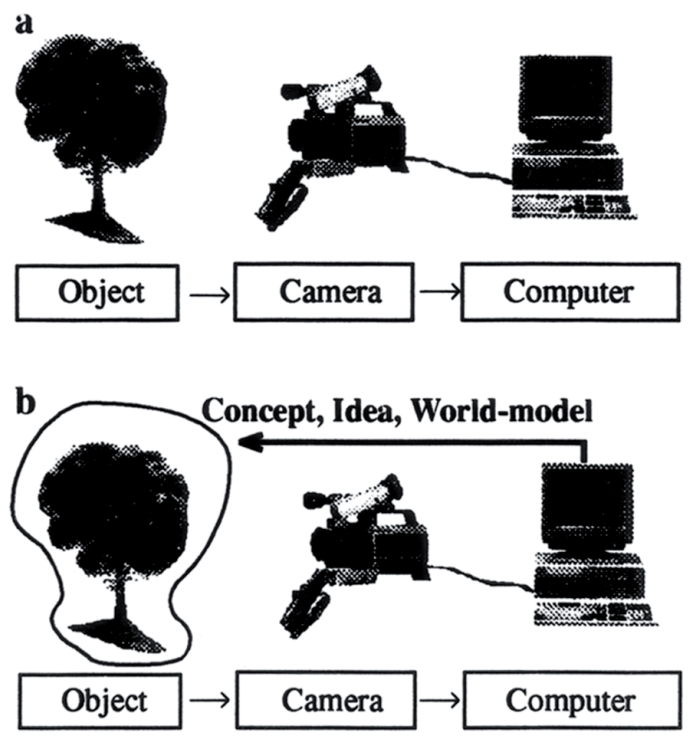

Figure 1. The computer-brain analogy

a) An object (tree) is filmed by a camera and the data are transferred to a computer where they are processed. b) In contrast to a computer, the human brain needs concepts to explain the sensory data, coming from sensory receptors, e.g., the eyes (camera).

This leads to the comparison between expected and real reality and includes the subjective experience which may be described in the terms: 'This occurs actually now'. In his Historical Anthropology Carl Friedrich von Weizsäcker claims, under the title 'As to the biology of subjects': "From empiricism one accepts sensory data as given [ . . ] it is not recognized that sensory data, already according to our biological constitution, can only be given under the prerequisite of a simultaneously perceived concept" (Weizsäcker 1982). 
The thinking of constructivism has - in opposition to empiricism - led to the concept that the term 'reality' has lost tremendously in its impact and unity, leading to a scientific climate in which we find ourselves in a 'realityrelativism' which may be characterized in terms like 'fictional reality' and internal 'reality-censorship'.

As a consequence, we can construe an interactive circuit in which interpretation of sensory data is possible only on the basis of conceptualizing hypotheses about possible realities; so to speak, nuclei of pre-categories which are - in a probational fashion - used to test the applicability of hypothetical world-models. The 'top-down components' might also be interpreted as the manifestation of a conctructivistic part within the neuronal basis of aisthesis. Thus, aisthesis incorporates at least two components: bottom-up analysis of data with top-down categorization.

The neurobiology of aisthesis, so to speak, the neuroscience of 'sensory cognition', represents an enterprise which has not yet reached a stable position of knowledge. It rather tries, within an iterative asymptotic approximation, to establish some innovative ideas how - due to a complex interaction of subcomponents within the plumbable brain - the subjective experiences in sensory cognition are generated (cf. Gregory 1970; 1980).

The interaction of top-down and bottom-up components of aisthesis under normal and altered circumstances is the main subject of our group's basic research in (psychiatric) cognitive neurosciences. Desimone has published a diagram (Fig. 2) in which the superimposition of bottom-up and top-down components is conceptualized as a manifestation of two streams of excitation, antagonizing each other (Gazzaniga et al. 2002). This function is related to a third component, relevant in processes of conceptualisation of 'reality', namely internal 'censorship'. This component is also attributed to plausibility control, to stabilization of internal data processing, if ambiguity and instability of perception occur, e.g., in emergency situations. It is attributed to a component which may work over incongruences in perception and thus may induce a predominance of fictional internal reality in comparison to the intricate, un-assimilated raw material of information about outer reality. This censorship is comparable to Gazzaniga's 'interpreter' (Gazzaniga 2000), a module which coordinates and integrates the computations (the 'votes') of other modules, and the result of such an 'interpreter'-related computation apparently has the subjective quality of being 'granted', being 'true', being 'real'. The function of this module is to stabilize 'reality fictions' and one may anticipate that especially this system is disturbed or, to an increased extent, 'vulnerable' in psychosis. To be more precise, in hallucinations, one may argue that it is the equilibrium between 'conceptualizations' and 'censorship' 
which is disturbed by relative censorship impairment. It appears to be possible to perform a type of illusion research on the basis of constructivism in which the fictional character of subjective reality can be elucidated and especially the perceptual disturbances in schizophrenic psychoses can be characterized. The basic concept herein is the contention, that perception does not represent one single procedure but is due to a complex interactive internal 'dialogue' between partial components of the system. And, interestingly, our research group has recently succeeded in demonstrating in such experiments that schizophrenia seems to be a result of a reduced ability to perform integrative aisthesis processes due to an inhibited connectivity between neuronal assemblies (Dima et al. 2011; Dima et al. 2010).

Concerning censorship, it has certainly great demands regarding long-term memory, since the question of what is 'plausible', 'sensible', 'biologically meaningful at present' can only be resolved on the basis of experience of failures and successes which are stored over longer periods. Thus, hippocampal structures may play a prominent role herein. Hippocampal structures may work as 'comparators' (Gray and Rawlins 1986) and calculate meaningful expectations in relation to sensory input.

Thus, the censorship systems described above may be characterized as such 'comparators'. This concept fits in with the idea that to have 'consciousness' means to 'compare' world models with actual (sensory or imagined) data (Emrich 1998) and further to have the opportunity to correct an obviously wrong intention - by a type of servo-mechanism - within the "last moment" (Gray 2004). Thus, an adequate definition of censorship may be the comparison of actual data with sets of acquired world models as the function of stabilizing 'reality fictions': and it is this that appears to be so highly developed in human mental life and which also appears to be so vulnerable and obviously impaired during psychotic states.

The scheme (adapted from Desimone 1995) shows the direction of (visual) bottom-up processing (right to left) from sensory cortex areas to higher associative cortex areas, especially in the temporal and prefrontal cortex. In contrast, top-down processing (left to right) is initiated by associative cortex areas, flowing in the opposite direction. 


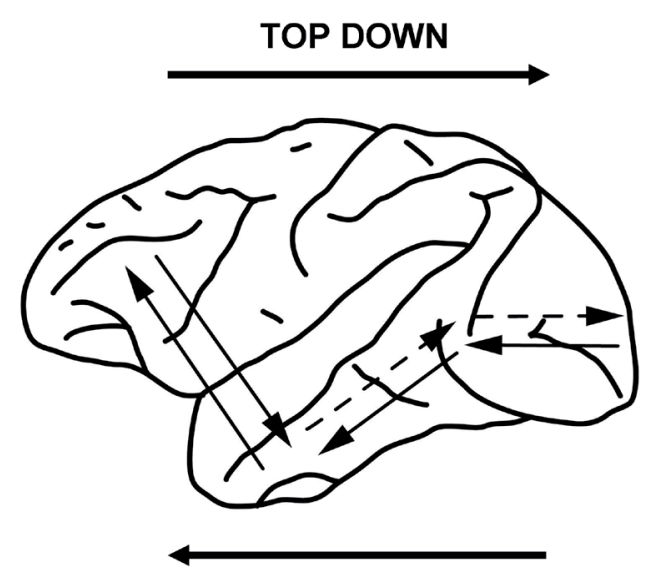

BOTTOM UP

Figure 2. Top-down and bottom-up processing in the human brain

\section{Three-Component Model of Perception / Aisthesis}

Taking into account all the considerations advanced above, one may propose a model describing three hypothetical components of aisthesis:

1. Sensuality ('bottom-up')

2. Constructivity ('top-down')

3. Censorship ('interpreter')

The three-component model of perception assumes that perception is principally made up of three functional domains: firstly, sensory input ('sensualistic' bottom-up component); secondly, the internal production of concepts ('constructivistic' top-down component); and thirdly, control ('censor' component). It also assumes that special interaction between these three components is responsible for a biologically fruitful and efficacious, conscious internal representation of the external world during perception and that the equilibrium between these three components may be disturbed in psychosis.

The constructivistic component can also be termed 'phantasy component', 'hypothesis-generating component', or 'conceptualization component'. Its representation in the present model takes the fact into account that processing of data is possible only on the basis of conceptualization which has to be applied to sensory data, before successful interpretation is possible. 
The 'censor' function can also be termed a 'correcting' function and may be qualified as a partially 'erasing' and partially 'suppressing' or 'rejecting' system. In the aisthesis process, a great difficulty arises: The more elaborate and complicated a 'private conscious world' is, the more prominent two problems become, which interact in an intrinsic antinomy: on the one hand, the fictional reality has to be so flexible that it can be adapted to the changing requirements of the external world; and, on the other hand, this private world has to be stabilized so far that it is not continuously in danger of losing its stability, i.e., to 'decompensate'. This means that both the flexibility of creativity and, on the other hand, the stability of the subjective world are required. To fulfil these two opposing requirements, intricate equilibria between neuronal networks have to be established. Disequilibrium of these neuronal components of the aisthesis-networks has been observed by our group in patients with schizophrenia and subjects under the condition of sleep deprivation and cannabinoid intoxication (Emrich et al. 1991; Emrich et al. 1997; Schneider et al. 1996; Schneider et al. 2002; Sternemann et al. 1997). Our group further demonstrated that, in schizophrenic patients, this is due to disturbed top-down connectivity, especially between the inferior parietal cortex and earlier sensory areas (Dima et al. 2009).

\section{Synaesthesia}

Synaesthesia appears to be a special case of aisthesis, which makes it possible to investigate the phenomenon more precisely in healthy subjects: normally synaesthesia does not impair every day life and therefore is not listed in common classification systems for neurological disorders (DSM-IV or ICD-10; Hubbard 2007).

Synaesthesia (Greek: syn = together; aesthesis = perception) represents, for everybody who comes in contact with this phenomenon for the first time, a very peculiar, astonishing and challenging process of intermingling of sensual percepts in which stimulation of one sensory modality or cognitive event leads to another internally generated perceptual experience. For example, hearing a tone or word may result in a subjective experience of seeing coloured shapes.

Synaesthetic experience can formally be divided into an inducing stimulus ('inducer') and an elicited perception ('concurrent') (Grossenbacher and Lovelace 2001). Most synaesthetes report to perceive synaesthesia for 'as long as they can remember' (Cytowic 2002). Its main characteristics are consistency (Baron-Cohen et al. 1987; Simner and Logie 2007) and automaticity (Mills et 
al. 1999). Consistency is actually used as a 'gold standard' in order to determine whether someone is a synaesthete or not (Baron-Cohen et al. 1987; Eagleman et al. 2007). There is evidence that conscious perception is necessary to elicit synaesthesia: Mattingley and colleagues showed that colour priming with letters is only possible when the letter is consciously perceived (Mattingley et al. 2001). Another study from Johnson et al. used the 'attentional blink' paradigm in which they found that some synaesthetes are able to report the colour of unconsciously perceived letters, whereas others are not (Johnson et al. 2007), underlining the importance of individual differences in synaesthesia (see also Laeng et al. 2004).

Although synaesthesia is believed to be a highly idiosyncratic phenomenon, there seem to be some general rules in synaesthetic perceptions, at least in grapheme-colour synaesthesia: frequently used letters are often coupled with frequently used colours (Simner et al. 2005) and tend to be brighter and more saturated (Beeli et al. 2007). These findings indicate that implicit learning mechanisms play a role in the development of inducerconcurrent couplings.

Recent field studies concerning the prevalence of synaesthesia revealed a prevalence of $4 \%$ (Simner et al. 2006). As synaesthesia runs more often within families (Baron-Cohen et al. 1996), there is evidence for a genetic component of synaesthesia and some gene regions have been recently identified to be involved in synaesthesia (Asher et al. 2009; Tomson et al. 2011).

\section{Models of synaesthesia}

In order to explain the neural mechanisms of synaesthesia particularly, two models gained recognition within the last years, which are not necessarily mutually exclusive. The model of 'local cross-activation', which is for instance supported by Hubbard and colleagues, proposes a direct linkage between an area referred to as 'visual word form area' and an adjacent region which has been shown to be involved in colour processing (hV4) in grapheme-colour synaesthesia (Hubbard et al. 2005). A possible reason for this linkage could be a failure of pruning in prenatal pathways (Hubbard and Ramachandran 2005; Maurer and Mondloch 2004). Other researchers suggested that synaesthesia might be due to a 'long-range disinhibited feedback' from a 'multisensory nexus' such as the temporo-parietal-occipital junction, so feedback connection that are usually inhibited (Grossenbacher and Lovelace 2001). 
Interestingly, synaesthetes commonly report that there is an apparent nonseparability of the semantic content of an inducer and the concurrent percept. This leads to the concept of 'hyperbinding' in synaesthesia (Esterman et al. 2006; Robertson 2003), which is in line with the concepts of cross-activation or disinhibited feedback: concurrent representation areas could be activated by cross-activation or disinhibited feedback mechanisms and additionally be bound to the inducer representation as the next step (Hubbard 2007).

\section{Neural correlates of synaesthesia: evidence from neurophysiological and neuroimaging studies}

Neuroimaging studies indicate that colour processing areas may play a role (as concurrent representation areas) at least in grapheme-colour synaesthesia, which is the most investigated form: Many studies revealed increased brain activation in these areas (Brang et al. 2010; Hubbard et al. 2005; Nunn et al. 2002), while others did not (Paulesu et al. 1995; Weiss et al. 2005; Rich et al. 2006). This has to be interpreted with caution. First, there is evidence that individual differences between synaesthetes determine the areas involved: A recent investigation in which synaesthetes were differentiated according to where they perceive their synaesthesia - projected to the written letter (projectors) or on an inner screen in their 'mind's eye' (associators) - found that visual areas showed increased activation only in projectors, while associators had additional activation and structural differences in limbic (especially hippocampal) brain areas (Rouw and Scholte 2010). Second, most investigations had only a rather small number of subjects $(<10)$ and it has been shown that, in neuroimaging studies, the results strongly depend on the number of investigated subjects (Thirion et al. 2007).

Further, there is strong evidence for an involvement of the parietal cortex in synaesthesia: Studies concerning different types of synaesthesia revealed increased parietal activation in synaesthetes compared to nonsynaesthetic controls when perceiving inducers (Rouw and Scholte 2010; Tang et al. 2008; Weiss et al. 2005; van Leeuwen et al. 2010), structural differences in the parietal cortex between synaesthetes and controls (Rouw and Scholte 2007; Weiss and Fink 2009) and a disruption of synaesthesia by TMS (Transcranial Magnetic Stimulation) over parietal sites (Esterman et al. 2006; Muggleton et al. 2007; Rothen et al. 2010). Recent investigations with fMRI by our own group (unpublished data) also found increased activation in the left inferior parietal cortex in grapheme-colour as well as auditoryvisual synaesthetes. As this area is a multimodal integration area which is also 
involved in non-synaesthetic binding (Robertson 2003), these findings speak in favour of an enhanced binding mechanism in synaesthesia. The parietal cortex may further be involved in synaesthesia as a sensory nexus area, leading to disinhibited feedback (Grossenbacher and Lovelace 2001). As this area is involved in auditory-visual, grapheme-colour and number-form (Tang et al. 2008) synaesthesia, it is likely that all types of synaesthesia share a common mechanism.

But also other multimodal areas have to be considered to be involved in synaesthetic binding: A neurophysiological investigation by our group in 1999 found in synaesthetes a more positive waveform of event related potential (ERPs) evoked by visual presentation of graphemes, especially over frontal and prefrontal sites (Schiltz et al. 1999), which was interpreted as prefrontal deactivation in synaesthesia. This was discussed as leading to increased distractibility, causing a leakage between modalities. Also, increased frontal brain activation due to synaesthesia has been detected using fMRI by other groups (Rouw and Scholte 2010; Tang et al. 2008; Paulesu et al. 1995). Therefore, prefrontal cortex areas seem to play a role in synaesthesia - although it still remains unclear which function they fulfil therein.

\section{Is synaesthesia a top-down phenomenon? Is Immanuel Kant's concept of synthesis erroneous?}

As we have pointed out above, synaesthesia can be regarded as a special case of aisthesis, an essential question is at which stage synaesthetic effects occur. Are they more bottom-up or top-down driven? At the first glance, synaesthesia seems to be a bottom-up phenomenon, as sensory perceptions are involved and mingled. But there is growing evidence for the involvement of top-down processes in synaesthesia. First, the synaesthetic concurrent depends on the interpretation of the inducer (Dixon et al. 2006; Bargary et al. 2009). Second, when grapheme-colour synaesthetes learn a new alphabet, the colour of a corresponding letter in the old alphabet transfers to the new letter (Mroczko et al. 2009) and is therefore rather concept-driven instead of depending on the letter's shape. Third, synaesthetic colours do not behave like real colours (van Leeuwen et al. 2010). All these findings speak in favour of the idea that synaesthesia is more a top-down phenomenon than an altered bottom-up processing. Further, the involvement of multimodal integration areas such as the parietal and the frontal cortex in synaesthesia, mentioned above, speaks against the idea of a direct (bottom-up) cross-activation 
of visual areas of concurrent representation. The parietal cortex is known to be involved in cross-modal integration (Calvert 2001), spatial processing (Colby and Goldberg 1999) and non-synaesthetic feature-binding (Robertson 2003), while the prefrontal cortex is thought to be the highest instance for executive functions, decision making and cognitive control (Miller 2000; Tanji and Hoshi 2001; Fuster 2001; Sakagami and Pan 2007). Further, both brain structures are part of a network involved in attention guidance and visuo-motor control (Grefkes et al. 2004; Astafiev et al. 2003; Corbetta and Shulman 2002). Therefore, both structures might work together during the formation of synaesthetic experience.

How might the concept of aisthesis help us to understand synaesthesia? If synaesthesia is a top-down phenomenon, it involves conceptualization. In synaesthetes, the concept of the inducer incorporates the concurrent dimension in addition to the normal dimensions of the concept. The bottomup analysis of the incoming signals activates the concept (top-down). The interpreter recognises the match, and the perception is formed through mingling the concept with the signals. As the concept incorporates the concurrent in addition, the overall perception is the fusion of both components (inducer and concurrent); the concurrent is additionally perceived.

The additional quality (e.g., colour) may be bound to the concept via a hyperbinding process (Esterman et al. 2006). Alternatively, it may be that synaesthetes afford additional complex categories (e.g., "A-red" in addition to "A" and "red"), forming an additional "reality-hypothesis". This would be in contrast to Kant's theory of synthesis, as it would mean that besides the pure concepts of reason there are also complex ones, as Hermann Schmitz has pointed out (Schmitz 1994) (personal communication, 2011). From this point of view, depicted within the scheme (Fig. 3), a modification of the concept of Desimone, categories in the sense of Kant appear, neurobiologically, to be not primary conditions of mind, but secondary conditions. Consequently, reality-hypotheses, appearing within the prefrontal cortex, are generated not as categorically partialized entities but primarily holistic ones (integral conceptual world), whereas the 'simple' categories might rather be represented by parietal cortex areas. One might further assume that, if a complex concept is activated, the corresponding 'simple' contexts are recalled via top-down processes.

But here the question remains, why synaesthetes have these enriched concepts. One explanation would be that it is a strategy to cope with problems with abstract thinking by concretizing abstract concepts with sensorial content like, for example colours, shapes, sounds. It is also thinkable that it is not a problem-solving strategy but a style of thinking: synaesthetes may be 
more sensorially orientated than the general population and use their senses to a higher degree in order to contextualize/make 'sense' of the world.

What does synaesthesia research tell us about aisthesis? Synaesthesia makes it very clear that aisthesis is not just a process of analysing sensory data and finding the corresponding concept to be able to interpret them but that concepts are also able to shape our perception.

\section{TOP DOWN}

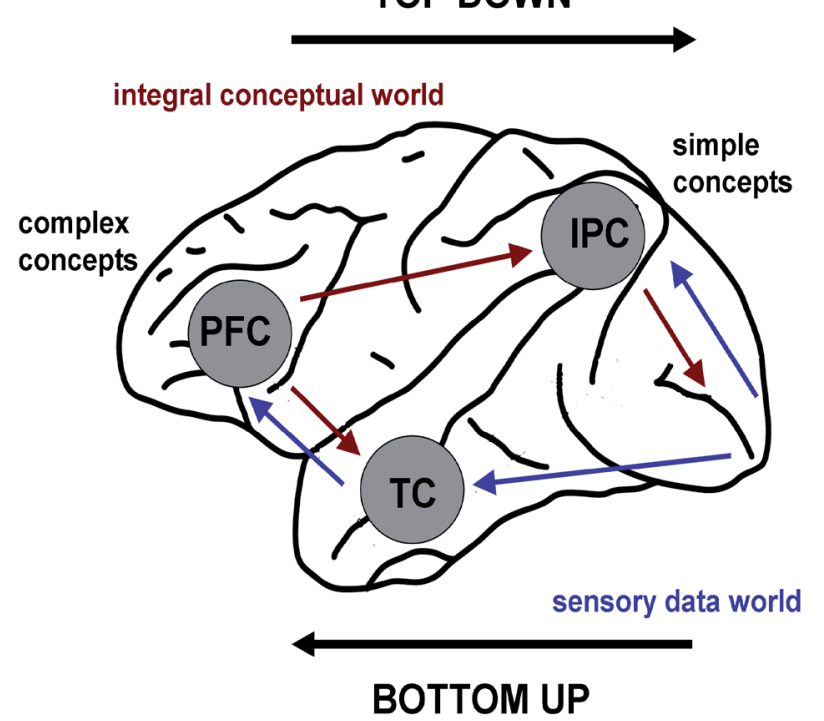

Figure 3. Advanced scheme of top-down and bottom-up processing

Sensory data (blue) coming from primary sensory cortex areas (here exemplarily from the occipital cortex) are processed to associative cortex regions, e.g., in the temporal cortex (TC), until they finally reach the prefrontal cortex (PFC). This leads to an excitation of complex concept-representations within the PFC, which are compared via top-down connections (red) to simple concepts, represented, e.g., by the inferior parietal cortex (IPC), and finally to sensory information. The world-model is represented by a network including PFC and IPC, which is related to sensory information by comparator functions, e.g., represented by limbic (temporal) structures. 


\section{Conclusion}

As a consequence of the current examinations, aisthesis appears as the result of complex, dynamical non-linear interaction of subcomponents of the brain, in which 'bottom-up' and 'top-down' components interact in a fashion yielding another component, a component of 'internal censorship', which apparently is partially hindered in conditions of psychoses and altered states of consciousness induced by drug intoxication or sleep deprivation. In contrast, in synaesthesia the top-down component may partially be enhanced, leading to additional complex context-categories and therefore to additional sensual experiences. The top-down component may be represented by a network of prefrontal and parietal structures which is also involved in attention guidance. This component may further represent a neuropsychological feature which also implies 'binding' properties.

\section{References}

Asher, Julian E., Janine A. Lamb, Denise Brocklebank, Jean-Baptiste Cazier, Elena Maestrini, Laura Addis, Mallika Sen, Simon Baron-Cohen and Anthony P. Monaco. 2009. "A whole-genome scan and fine-mapping linkage study of auditory-visual synesthesia reveals evidence of linkage to chromosomes 2q24, 5q33, 6p12, and 12p12." The American Journal of Human Genetics 84: 279-285.

Astafiev, Serguei V., Gordon L. Shulman, Christine M. Stanley, Abraham Z. Snyder, David C. Van Essen and Maurizio Corbetta. 2003. "Functional organization of human intraparietal and frontal cortex for attending, looking, and pointing." Journal of Neuroscience 23: 4689-4699.

Bargary, Gary, Kylie J. Barnett, Kevin J. Mitchell and Fiona N. Newell. 2009.

"Colored-speech synaesthesia is triggered by multisensory, not unisensory, perception." Psychological science: a Journal of the American Psychological Society / APS 20: 529-533.

Baron-Cohen, Simon, Lucy Burt, Fiona Smith-Laittan, John Harrison and Patrick Bolton. 1996. "Synaesthesia: prevalence and familiality." Perception 25: 1073-1079.

Baron-Cohen, Simon, Maria A. Wyke and Colin Binnie. 1987. "Hearing words and seeing colours: an experimental investigation of a case of synaesthesia." Perception 16: 761-767.

Beeli, Gian, Michaela Esslen and Lutz Jäncke. 2007. "Frequency correlates in grapheme-color synaesthesia." Psychological Science 18: 788-792. 
Brang, David, Edward M. Hubbard, Seana Coulson, Minxiong Huang and Vilayanur S. Ramachandran. 2010. "Magnetoencephalography reveals early activation of V4 in grapheme-color synesthesia." Neuroimage 53: 268-274.

Calvert, Gemma A. 2001. "Crossmodal processing in the human brain: insights from functional neuroimaging studies." Cerebral Cortex 11: 1110-1123.

Colby, Carol L. and Michael E. Goldberg. 1999. "Space and attention in parietal cortex." Annual Review of Neuroscience 22: 319-349.

Corbetta, Maurizio and Gordon L. Shulman. 2002. "Control of goal-directed and stimulus-driven attention in the brain." Nature Reviews Neuroscience 3: 201215.

Cytowic, Richard E. 2002. Synesthesia: A Union of the Senses. (2nd ed.) Cambridge, Massachussetts; London, England: MIT Press.

Desimone, Robert, Earl K. Miller, Leonardo Chelazzi and Andreas Lueschow. 1995. Multiple Memory Systems in the Visual Cortex. In Michael Gazzaniga (ed.). The Cognitive Neurosciences, 475-486. Cambridge, Massachusetts: MIT Press.

Dima, Danai, Detlef E. Dietrich, Wolfgang Dillo and Hinderk M. Emrich. 2010. "Impaired top-down processes in schizophrenia: a DCM study of ERPs." Neuroimage 52: 824-832.

Dima, Danai, Wolfgang Dillo, Catharina Bonnemann, Hinderk M. Emrich and Detlef E. Dietrich. 2011. "Reduced P300 and P600 amplitude in the hollow-mask illusion in patients with schizophrenia." Psychiatry Research 191: 145-151.

Dima, Danai, Jonathan P. Roiser, Detlef E. Dietrich, Catharina Bonnemann, Heinrich Lanfermann, Hinderk M. Emrich, H. M. and Wolfgang Dillo. 2009. "Understanding why patients with schizophrenia do not perceive the hollowmask illusion using dynamic causal modelling." Neuroimage 46: 1180-1186.

Dixon, Mike J., Daniel Smilek, Patricia L. Duffy, Mark P. Zanna and Philip M. Merikle. 2006. "The role of meaning in grapheme-colour synaesthesia." Cortex 42: 243-252.

Eagleman, David M., Arielle D. Kagan, Stephanie S. Nelson, Deepak Sagaram and Anand K. Sarma. 2007. "A standardized test battery for the study of synesthesia." Journal of Neuroscience Methods 159: 139-145.

Emrich, Hinderk M. 1998. "Wahrnehmung, Mimesis und Bewußtsein" [Perception, mimesis and consciousness]. Fortschritte der Neurologie Psychiatrie 66: 8493.

Emrich, Hinderk M., F. Markus Leweke and Udo Schneider. 1997. "Towards a cannabinoid hypothesis of schizophrenia: cognitive impairments due to dysregulation of the endogenous cannabinoid system." Pharmacology Biochemistry and Behavior 56: 803-807.

Emrich, Hinderk M., Marcus M. Weber, A. Wendl, Josef Zihl, L. Vonmeyer and W. H. Hanisch. 1991. "Reduced binocular depth inversion as an indicator of cannabis-induced censorship impairment." Pharmacology Biochemistry and Behavior 40: 689-690. 
Esterman, Michael, Timothy Verstynen, Richard B. Ivry and Lynn C. Robertson. 2006. "Coming unbound: disrupting automatic integration of synesthetic color and graphemes by transcranial magnetic stimulation of the right parietal lobe." Journal of Cognitive Neuroscience 18: 1570-1576.

Fuster, Joaquin M. 2001. "The prefrontal cortex - an update: time is of the essence." Neuron 30: 319-333.

Gazzaniga, Michael S. 2000. "Cerebral specialization and interhemispheric communication: does the corpus callosum enable the human condition?" Brain 123(7): 1293-1326.

Gazzaniga, Michael S., Richard B. Ivry and George R. Mangun. 2002. Neuroscience: The Biology of the Mind. (2nd ed.). New York: W. W. Norton.

Gray, Jeffrey A. 2004. Consciousness: Creeping up on the Hard Problem. (1 ed.) Oxford University Press.

Gray, Jeffrey A. and John Nicholas P. Rawlins. 1986. Comparator and buffer memory: An attempt to integrate two models of hippocampal functions. In Robert L. Isaacson and Karl H. Pribram (eds.), The hippocampus (4 ed.). New York: Plenum.

Grefkes, Christian, Afra Ritzl, Karl Zilles and Gereon R. Fink. 2004. "Human medial intraparietal cortex subserves visuomotor coordinate transformation." Neuroimage 23: 1494-1506.

Gregory, Richard L. 1970. The intelligent eye. London: Weidenfeld \& Nicolson.

Gregory, Richard L. 1980. "Perceptions as hypotheses." Philosophical Transactions of the Royal Society, B. 290: 181-197

Grossenbacher, Peter G. and Christopher T. Lovelace. 2001. "Mechanisms of synesthesia: cognitive and physiological constraints." Trends in Cognitive Sciences 5: 36-41.

Hubbard, Edward M. 2007. "Neurophysiology of synesthesia." Current Psychiatry Reports 9: 193-199.

Hubbard, Edward M., A. Cyrus Arman, Vilayanur S. Ramachandran and Geoffrey M. Boynton. 2005. "Individual differences among grapheme-color synesthetes: brain-behavior correlations." Neuron 45: 975-985.

Hubbard, Edward M. and Vilayanur S. Ramachandran. 2005. "Neurocognitive mechanisms of synesthesia." Neuron 48: 509-520.

Hubel, David H. and Torsten N. Wiesel. 1979. "Brain mechanisms of vision." Scientific American 241: 150-162.

Johnson, Addie, Marieke Jepma and Ritske R. De Jong. 2007. "Colours sometimes count: awareness and bidirecionality in grapheme-colour synaesthesia." Quarterly Journal of Experimental Psychology (Colchester) 60: 1406-1422.

Kant, Immanuel 1974. Kritik der reinen Vernunft. Frankfurt am Main: Suhrkamp.

Laeng, Bruno, Frode Svartdal and Hella Oelmann. 2004. "Does color synesthesia pose a paradox for early-selection theories of attention?" Psychological Science 15: 277-281. 
Mattingley, Jason B., Anina N. Rich, Greg Yelland and John L. Bradshaw. 2001. "Unconscious priming eliminates automatic binding of colour and alphanumeric form in synaesthesia." Nature 410: 580-582.

Maurer, Daphne and Catherine J. Mondloch. 2004. Neonatal synesthesia: A reevaluation. In Lynn Robertson and Noam Sagiv (eds.), Synesthesia: Perspectives from Cognitive Neuroscience, 193-213. Oxford Univerity Press.

Merzenich, Michael M. and John F. Brugge. 1973. "Representation of the cochlear partition of the superior temporal plane of the macaque monkey." Brain Research 50: 275-296.

Miller, Earl K. 2000. "The prefrontal cortex and cognitive control." Nature Reviews Neuroscience 1: 59-65.

Mills, Carol Bergfeld, Edith Howell Boteler and Glenda K. Oliver. 1999. "Digit synaesthesia: Acase study using a Stroop-type test." Cognitive Neuropsychology 16: 181-191.

Mroczko, Aleksandra, Thomas Metzinger, Wolf Singer and Danko Nikolić. 2009. "Immediate transfer of synesthesia to a novel inducer." Journal of Vision 9: $25,1-8$.

Muggleton, Neil, Elias Tsakanikos, Vincent Walsh and Jamie Ward. 2007. "Disruption of synaesthesia following TMS of the right posterior parietal cortex." Neuropsychologia 45: 1582-1585.

Nunn, J. A., L. J. Gregory, M. Brammer, S. C. R. Williams, D. M. Parslow, M. J. Morgan, R. G. Morris, E. T. Bullmore, S. Baron-Cohen and J. A. Gray. 2002. "Functional magnetic resonance imaging of synesthesia: activation of V4/V8 by spoken words." Nature Neuroscience 5: 371-375.

Paulesu, Eraldo, John Harrison, Simon Baron-Cohen, J. D. Watson, L. Goldstein, J. Heather, R. S. J. Frackowiak and C. D. Frith. 1995. "The physiology of coloured hearing. A PET activation study of colour-word synaesthesia." Brain 118(3): 661-676.

Prinz, Wolfgang. 2006. "What re-enactment earns us." Cortex 42: 515-517.

Rich, Anina N., Mark A. Williams, Aina Puce, Ari Syngeniotis, Matthew A. Howard, Francis McGlone and Jason B. Mattingley. 2006. "Neural correlates of imagined and synaesthetic colours." Neuropsychologia 44: 2918-2925.

Robertson, Lynn C. 2003. "Binding, spatial attention and perceptual awareness." Nature Reviews Neuroscience 4: 93-102.

Rothen, Nicolas, Thomas Nyffeler, Roman von Wartburg, René Müri and Beat Meier. 2010. "Parieto-occipital suppression eliminates implicit bidirectionality in grapheme-colour synaesthesia." Neuropsychologia 48: 3482-3487.

Rouw, Romke and H. Steven Scholte. 2007. "Increased structural connectivity in grapheme-color synesthesia." Nature Neuroscience 10: 792-797.

Rouw, Romke and H. Steven Scholte. 2010. "Neural basis of individual differences in synesthetic experiences." Journal of Neuroscience 30: 6205-6213.

Sakagami, Masamichi and Xiaochuan Pan. 2007. "Functional role of the ventrolateral prefrontal cortex in decision making." Current Opinion in Neurobiology 17: 228-233. 
Schiltz, Kolja, Karen Trocha, Bernardina M. Wieringa, Hinderk M. Emrich, Sönke Johannes and Thomas F. Münte. 1999. "Neurophysiological aspects of synesthetic experience." The Journal of Neuropsychiatry and Clinical Neurosciences 11: 58-65.

Schmitz, Hermann. 1994. Neue Grundlagen der Erkenntnistheorie. Bouvier.

Schneider, U., M. Borsutzky, J. Seifert, F. M. Leweke, T. J. Huber, J. D. Rollnik and H. M. Emrich. 2002. "Reduced binocular depth inversion in schizophrenic patients." Schizophrenia Research 53: 101-108.

Schneider, U., F. M. Leweke, W. Niemcyzk, U. Sternemann, M. Bevilacqua and H. M. Emrich. 1996. "Impaired binocular depth inversion in patients with alcohol withdrawal." Journal of Psychiatric Research 30: 469-474.

Simner, Julia and Robert H. Logie. 2007. "Synaesthetic consistency spans decades in a lexical-gustatory synaesthete." Neurocase 13: 358-365.

Simner, Julia, Catherine Mulvenna, Noam Sagiv, Elias Tsakanikos, Sarah A. Witherby, Christine Fraser, Kirsten Scott and Jamie Ward. 2006. "Synaesthesia: the prevalence of atypical cross-modal experiences." Perception 35: 1024-1033.

Simner, Julia, Jamie Ward, Monika Lanz, Ashok Jansari, Krist Noonan, Louise Glover and David A. Oakley. 2005. "Non-random associations of graphemes to colours in synaesthetic and non-synaesthetic populations." Cognitive Neuropsychology 22: 1069-1085.

Sternemann, U., Udo Schneider, F. Markus Leweke, C. M. Bevilacqua, Detlef E. Dietrich and Hinderk M. Emrich. 1997. "Propsychotische veränderung der binokulären tiefeninversion durch schlafentzug" [Pro-psychotic change of binocular depth inversion by sleep deprivation]. Nervenarzt 68: 593-596.

Tang, Joey, Jamie Ward and Brian Butterworth. 2008. "Number forms in the brain." Journal of Cognitive Neuroscience 20: 1547-1556.

Tanji, Jun and Eiji Hoshi. 2001. "Behavioral planning in the prefrontal cortex." Current Opinion in Neurobiology 11: 164-170.

Thirion, Bertrand, Philippe Pinel, Sébastien Mériaux, Alexis Roche, Stanislas Dehaene and Jean-Baptiste Poline. 2007. "Analysis of a large fMRI cohort: Statistical and methodological issues for group analyses." Neuroimage 35: 105-120.

Tomson, Steffie N., Nili Avidan, Kwanghyuk Lee, Anand K. Sarma, Rejnal Tushe, Dianna M. Milewicz, Molly Bray, Suzanne M. Leal, and David M. Eagleman. 2011. "The genetics of colored sequence synesthesia: Suggestive evidence of linkage to 16q and genetic heterogeneity for the condition." Behavioural Brain Research 223: 48-52.

van Leeuwen, Tessa M., Karl Magnus Petersson and Peter Hagoort. 2010. "Synaesthetic colour in the brain: beyond colour areas. A functional magnetic resonance imaging study of synaesthetes and matched controls." PLOS ONE 5: e12074.

von Weizsäcker, Carl Friedrich. 1982. Zur Biologie des Subjekts. In Der Garten des Menschlichen, 169-224. München, Wien: Carl Hanser Verlag. 
Weiss, Peter H. and Gereon R. Fink. 2009. "Grapheme-colour synaesthetes show increased grey matter volumes of parietal and fusiform cortex." Brain 132: 65-70.

Weiss, Peter H., Karl Zillesand and Gereon R. Fink. 2005. "When visual perception causes feeling: enhanced cross-modal processing in grapheme-color synesthesia." Neuroimage 28: 859-868.

Welsch, Wolfgang. 1992. Die Aktualität des Ästhetischen. In Die Aktualität des Ästhetischen. München: Fink.

Wertheimer, Max. 1938. Gestalt Psychology. In Willis D. Ellis (ed.), Source Book of Gestalt Psychology. New York: Harcourt, Brace and Co. 